\title{
Biomimetic Anti-adhesive Surface Micro-structures of Electrosurgical Knife Fabricated by Fibre Laser
}

\author{
Chen $\mathrm{Li}^{1}$, Li-jun Yang ${ }^{1}$, Cheng-cheng Yan $^{1}$, Wei Chen ${ }^{1}$, Guang-hua Cheng ${ }^{2}$ \\ ${ }^{1}$ College of Mechanical and Electrical Engineering, Shaanxi University of Science and Technology, \\ Xi'an 710021, Shaanxi, China \\ E-mail: lichen@sust.edu.cn \\ 2 State Key Laboratory of Transient Optics and Photonics, Xi'an Institute of Optics and Precision \\ Mechanics, CAS, Xi'an 710119, Shaanxi, China
}

\begin{abstract}
Electrosurgical knife is the most common invasive surgical instrument in a cutting and hemostasis process, which easily leads the overheated tissues to pyrolysis, eschar and adhesion on the knife surface. In order to minimize the adhesion of the overheated tissues, we investigated and fabricated the surface micro-structures on the blade by fiber laser inspired by shark's skin. The wettability of the blade surfaces with different bionic shark skins were measured by the contact angles, revealing the surface wettability effect. Frictional coefficients between the blade surfaces with different bionic shark skins and pork liver tissues are respectively measured by friction test machine. After cutting the pork liver, the adhesive tissue mass on the electrosurgical knives are weighed to study the antiadhesion. The relationship between adhesive mass, wettability and frictional coefficients on the bionic shark skins are analyzed in the orthogonal experimental method. The experimental results show that the bionic shark skin on the blades can effectively reduce the adhesive tissues.
\end{abstract}

DOI: $10.2961 /$ jlmn.2018.03.0028

Keywords: laser precision microfabrication, anti-adhesion, biomimetic shark skin, micro-structure, stainless steel 316L.

\section{Introduction}

Electrosurgery is broadly used in a wide variety of surgical procedures, and electrosurgical knives are the most common invasive surgical instruments in a cutting and hemostasis process [1]. In surgery, the surgeon controls the radio-frequency electrical energy to the blade to generate heat and cause hemostasis as tissue is cut, which easily leads the overheated tissues to pyrolysis, eschar and adhesion on the knife surface. Although electrosurgical knives have proven effective for minimizing bleeding during surgery, one problem is that the overheated tissue sticking to the blade reduces cutting efficiency and ultimately requires the replacement of the blade [2]. Traditional methods to overcoming this problem rely on simple coating technology [3] or edge shape optimization technology [4], which are difficult to satisfy the requirements of minimally invasive medical devices.

In recent years, the surface modification of the electrosurgical knives to reduce tissue adhesion has attracted the attention [5, 6]. Zhang et al. proposed the micro/nanostructures on the knife surface, filled with biocompatible lubricating liquids (e.g. water, silicone oil, polyethylene glycol and polyethylene oxide) to reduce adhesion [5]. Lin et al. used femtosecond laser to produce micro/nanostructures on the electrode surface, which can effectively avoid tissue damage [6]. However, these methods involved the complex or expensive equipments, which limited the applications.

Inspired by the remarkable nature example of the shark skin, the excellent performances of drag reduction and antifouling induced by its distinctive surface morphology with hierarchical micro/nano-structures attracted worldwide attention [7-10], as shown in Fig.1. Chen et al. replicated the outer surface of shark skin in a large area fabricated by a bio-replicated micro-imprinting approach and the test showed that the drag reduction and anti-fouling function of the coating surface were excellent [9]. Fu et al. fabricated the shark skin replica by the micro molding technique and improve the wettability function [10]. Because of the shark skin's functional performances of drag reduction and antifouling, we investigate and fabricate the biomimetic antiadhesion surface micro structures on the electrosurgical blades in this paper. Compared with the expensive ultrafast laser including femtosecond and picosecond laser, longpulse fiber lasers including the millisecond and microsecond laser are cheaper and widely used in the industry. So this work focused on the biomimetic anti-adhesion surface structures fabricated by the long-pulse fiber laser.

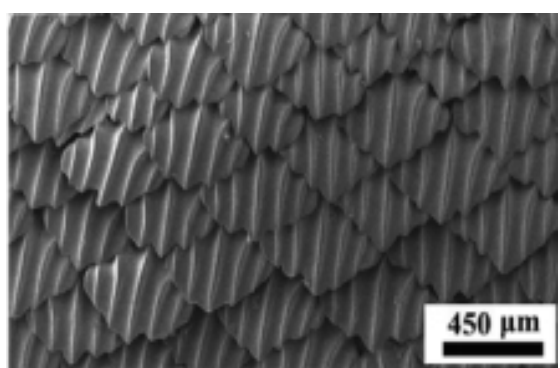

Fig. 1 SEM images of the shark skin's surface morphology [7] .

\section{Experiment}




\subsection{Materials}

The experiments detailed in this paper were performed on commercially available stainless steel 316L sheets with thicknesses of $0.64 \mathrm{~mm}$ and electrosurgical blades made of stainless steel 316L, respectively. Before and after laser radiation, the samples were cleaned ultrasonically with acetone, ethanol and deionized water successively.

\subsection{Surface laser irradiation}

Hierarchical structures on the sample surface were fabricated using a SPI fiber laser (SPI-100C) system at the wavelength of $1070 \mathrm{~nm}$ with the pulse width of $500 \mu \mathrm{s}$. The sample was mounted on a computer-controlled XYZ motion platform. The laser micromachining system used in the experiment mainly includes a laser source, an optical path system, and a motion platform, as shown in Fig. 2. During the laser processing, the surface micro-structures are fabricated directly by controlling the motion platform with the sample. The sample was irradiated by the laser beam normal to the surface, with focus spot of about $18 \mu \mathrm{m}$ diameter. The scanning speed of $0.5 \sim 5 \mathrm{~m} / \mathrm{min}$ and repetition rate of $1000 \mathrm{~Hz}$ were used. The fluences in the range of 2000 20000 J/cm² were applied separately to the sample 316L sheets and electrosurgical blades. Arrays of microgrooves were directly written on the sample surface.

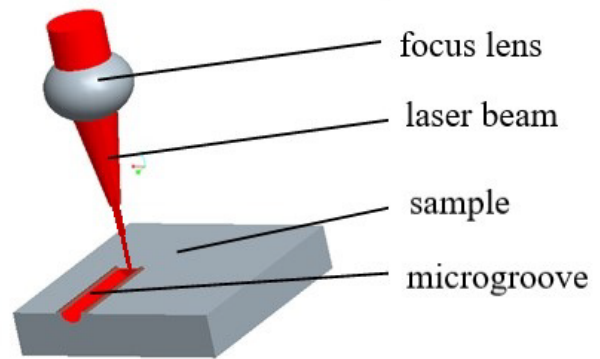

Fig. 2 Schematic diagram of the laser micromachining system.

In Fig. 1, the surface of shark skin is composed of tiny diamond-shaped scales with small riblets aligned in the direction of fluid flow, which contributes to the drag reduction and antifouling. Similar to the distinctive surface morphology of shark skin, we built the surface structure model, shown in Fig. 3. In the model, lots of same square scales with length $\mathrm{L}$ built up the surface structures and the riblets with pitch $\mathrm{D}$ in the direction along fluid flow cover the scale surface. According to the model, the bionic shark skin is directly written on the samples by fiber laser pulses. Fig. 4 shows a result of bionic shark skin surface fabricated by laser on stainless steel. Fig. 5 shows the depth of the grooves fabricated by the laser at the given laser power, which made up the bionic shark skins.

To find the optimal structural parameters, orthogonal experimental methods were used [11]. 9 blades with different structures (in different parameters: P, D, L) are fabricated, the structural parameters are shown in the table 1 . Another smooth blade without bionic shark skin was used as a reference in the following experiments.

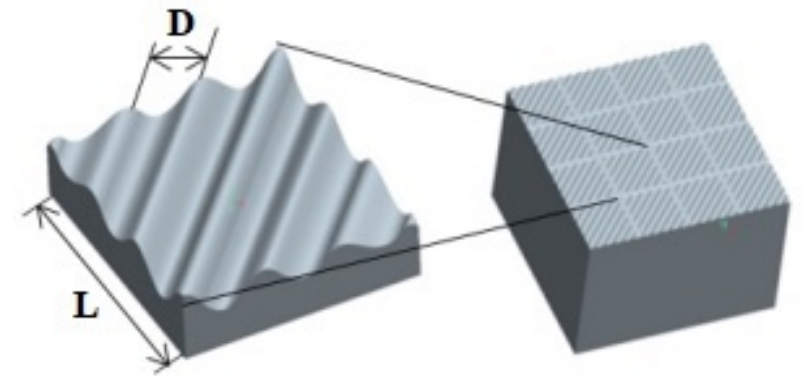

Fig. 3 Model of bionic shark skin.

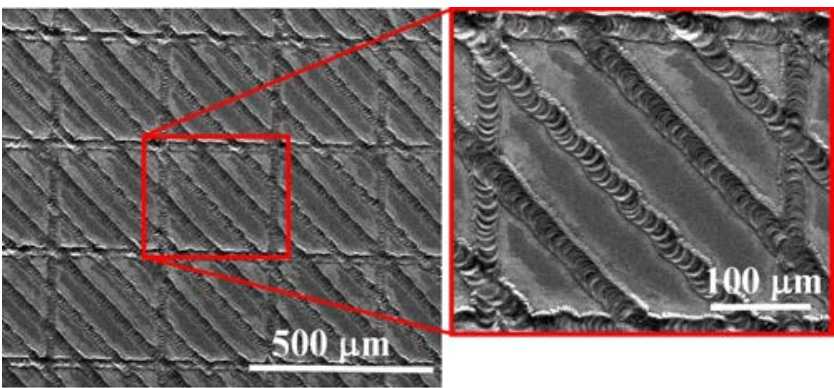

Fig. 4 SEM image of bionic shark skin surface fabricated by laser.

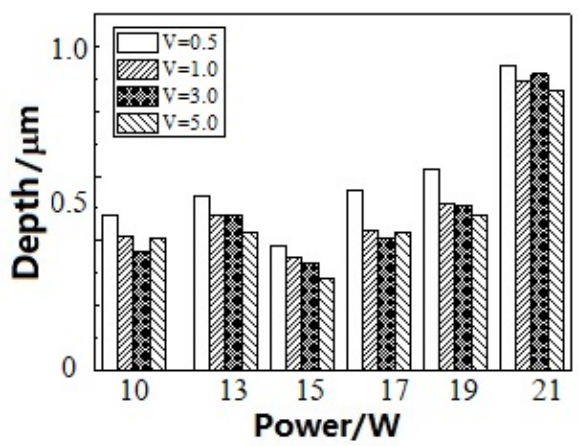

Fig. 5 The depth of the grooves fabricated by the laser at the given laser power.

Table 1 The experimental parameters of bionic shark skin.

\begin{tabular}{cccc}
\hline Sample No. & $\mathbf{P} / \mathbf{W}$ & $\mathbf{D} / \boldsymbol{\mu \mathbf { m }}$ & $\mathbf{L} / \boldsymbol{\mu \mathbf { m }}$ \\
\hline 1 & 15 & 150 & 600 \\
2 & 15 & 300 & 800 \\
3 & 15 & 450 & 1000 \\
4 & 21 & 150 & 800 \\
5 & 21 & 300 & 1000 \\
6 & 21 & 450 & 600 \\
7 & 18 & 150 & 1000 \\
8 & 18 & 300 & 600 \\
9 & 18 & 450 & 800 \\
\hline
\end{tabular}

\subsection{Surface measurement and test}

After different bionic shark skins were fabricated on 9 blades, the surface properties of bionic shark skins were measured and tested. At first, scanning electron microscopy (SEM, FEI Q45) and optical microscopy were used to study the surface morphology of bionic shark skins. Second, surface wettability of samples was studied by measuring 
the contact angle under ambient conditions. The friction coefficients of samples were measured by friction and wear testing machine under ambient conditions. At last, after the blades with bionic shark skins were tested to cut the pig's liver, the mass of adhesion tissues on the blades were weighed to study the anti-adhesion.

\section{Results and discussion}

The tissue adhesion on the blade is related to several factors, such as blade materials, blade shape, surface wettability, friction coefficients and so on. This paper mainly focuses on the effects of surface wettability and friction coefficient on the anti-adhesion performance on the commercial electrosurgical blades made of stainless steel. The effects of surface wettability and friction coefficient were analyzed and discussed separately in the following.

\subsection{Surface wettability effect}

The samples ( 9 blades with different structures in table 1) were left in air, under ambient conditions before studying their surface wettability by contact angle meter (Dataphyscs, OCA20). A $5 \mu \mathrm{L}$ droplet of deionized water was dispensed onto the sample surface using a syringe while an image was captured by a camera combined with a $6 \times$ magnification system. The contact angle was then determined by analyzing the droplet images using the software SCA20. The contact angles on each sample were measured in several times and the average values of contact angles were obtained, shown in Fig. 6.

In Fig. 6, the contact angle on the smooth original sample was $68^{\circ}$, which is smaller than the other samples with bionic shark skin. So biomimetic shark skin can increase the contact angle of the knife surfaces and reduce the hydrophilicity. The contact angle can be increased by up to $21 \%$.

In order to find the role of structures parameters (P, D, $\mathrm{L})$ in the surface wettability, orthogonal experimental methods were used to analyze the contact angle results in Fig. 6. Orthogonal experimental results are shown in table 2. For example, in the case of parameter $\mathrm{P}$ (the left column in Table 2), $K_{1}, K_{2}$ and $K_{3}$ are defined as follow. $K_{1}, K_{2}$ and $\mathrm{K}_{3}$ are sum of the contact angles on the samples at $\mathrm{P}=15$, 21 and $18 \mathrm{~W}$ (shown in Table 1), respectively. Then $\mathrm{k}_{1}=\mathrm{K}_{1} / 3=82.67, \mathrm{k}_{2}=\mathrm{K}_{2} / 3=82.44$, and $\mathrm{k}_{3}=\mathrm{K}_{3} / 3=81.18$. At last, $\mathrm{R}_{\mathrm{P}}=$ range of $\left(\mathrm{k}_{1}, \mathrm{k}_{2}, \mathrm{k}_{3}\right)=1.49$. Similarly, $\mathrm{R}_{\mathrm{D}}=$ range of $\left(\mathrm{k}_{1}\right.$, $\left.k_{2}, k_{3}\right)=0.22$ for parameter $D ; R_{L}=$ range of $\left(k_{1}, k_{2}, k_{3}\right)$ $=2.69$ for parameter $\mathrm{L}$. From table $2, \mathrm{R}_{\mathrm{L}}>\mathrm{R}_{\mathrm{P}}>\mathrm{R}_{\mathrm{D}}$, implying that structural parameters affecting wettability are as following: Scale length $(\mathrm{L})>$ Laser power $(\mathrm{P})>$ Riblets pitch (D). So the macro structures with scale length $L$ play the most important role in surface wettability.

The mechanism of surface structures affecting the contact angle can be explained by Cassie-Baxter model [12]:

$$
\cos \left(\theta^{*}\right)=r_{f} f \cos \left(\theta_{\mathrm{Y}}\right)+f-1
$$

where $\theta^{*}$ is the apparent contact angle which corresponds to the stable equilibrium state, $\theta_{\mathrm{Y}}$ is the Young contact angle as defined for an ideal surface, $\mathrm{r}_{\mathrm{f}}$ is the roughness ratio of the wet surface area and $f$ is the fraction of solid surface area wet by the liquid. The structural parameters $(\mathrm{P}, \mathrm{D}, \mathrm{L})$ of bionic shark skin change $\mathrm{r}_{\mathrm{f}}$ and $\mathrm{f}$ in the Cassie-Baxter model, resulting in the increased contact angle.

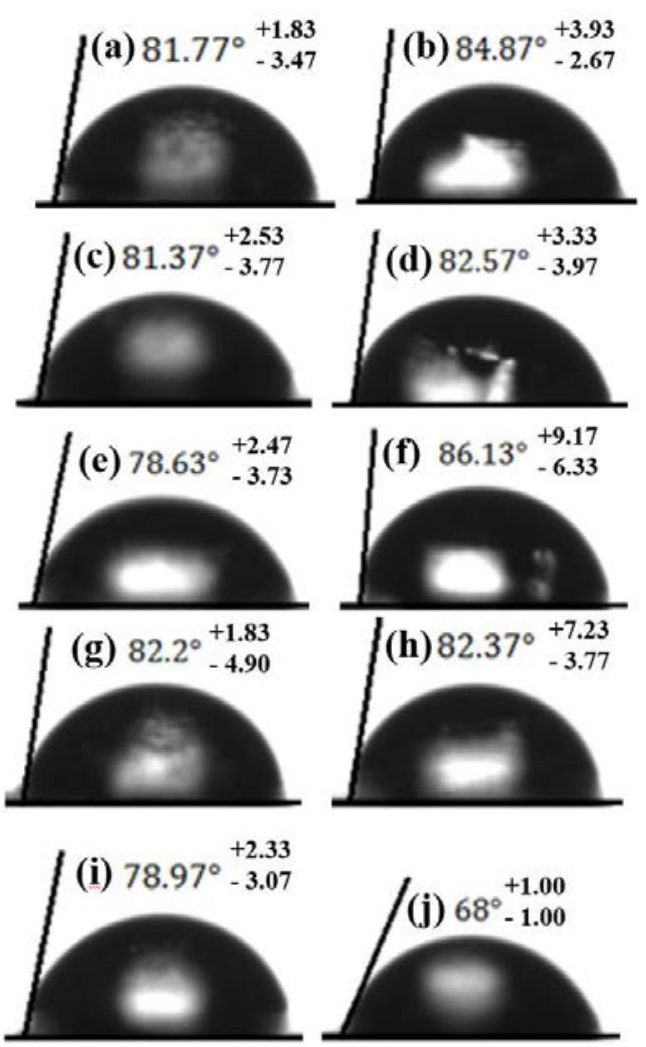

Fig. 6 Contact angles of sample surfaces: (a) Sample No.1; (b) Sample No.2; (c) Sample No.3; (d) Sample No.4; (e) Sample No.5; (f) Sample No.6; (g) Sample No.7; (h) Sample No.8; (i) Sample No.9; (j) Original sample without bionic shark skin.

Table 2 Orthogonal experiment result of surface wettability

\begin{tabular}{c|ccc}
\hline & $\mathrm{P} / \mathrm{W}$ & $\mathrm{D} / \mu \mathrm{m}$ & $\mathrm{L} / \mu \mathrm{m}$ \\
\hline $\mathrm{K}_{1}$ & 248.01 & 246.54 & 250.27 \\
$\mathrm{~K}_{2}$ & 247.33 & 245.87 & 249.81 \\
$\mathrm{~K}_{3}$ & 243.54 & 246.47 & 242.2 \\
$\mathrm{k}_{1}\left(=\mathrm{K}_{1} / 3\right)$ & 82.67 & 82.18 & 83.42 \\
$\mathrm{k}_{2}\left(=\mathrm{K}_{2} / 3\right)$ & 82.44 & 81.96 & 83.27 \\
$\mathrm{k}_{3}\left(=\mathrm{K}_{3} / 3\right)$ & 81.18 & 82.16 & 80.73 \\
$\mathrm{R}_{(\mathrm{P}, \mathrm{D}, \mathrm{L})}$ & 1.49 & 0.22 & 2.69 \\
\hline
\end{tabular}

\subsection{Friction test}

To study the friction effect on the anti-adhesion performance, the friction coefficients between the blade surfaces and pig's liver slices were measured using the vertical universal friction and wear testing machine (MMW-1). In the machine, the pig's liver was cut to the disc-shaped slice with the diameter of $ø 45 \mathrm{~mm}$ and thickness of $2 \mathrm{~mm}$, which was fixed on a metal substrate. The blade tip was cut into the rectangular bar of $5 \mathrm{~mm}$ length, that was fixed on a pin in the machine. In the friction test, the pressure force between blade surface and liver slice is set to $10 \mathrm{~N}$, and rotation speed of the blade tip on the liver slice is set to 10 
$\mathrm{r} / \mathrm{min}$. The friction experiments were carried out at room temperature under ambient conditions. The friction coefficient of each sample was measured at least 3 times to obtain the average value as the result. The friction coefficients of all the blades with bionic shark skins in table 1 were shown in table 3.

Table 3 Friction coefficients of samples with bionic shark skins.

\begin{tabular}{c|ccc}
\hline Sample No. & $\mathbf{1}$ & 2 & 3 \\
\hline $\begin{array}{c}\text { Friction } \\
\text { coefficient f }\end{array}$ & $0.21_{-0.058}^{+0.088}$ & $0.20_{-0.066}^{+0.032}$ & $0.22_{-0.077}^{+0.050}$ \\
\hline Sample No. & $\mathbf{4}$ & $\mathbf{5}$ & $\mathbf{6}$ \\
\hline $\begin{array}{c}\text { Friction } \\
\text { coefficient f }\end{array}$ & $0.23_{-0.114}^{+0.044}$ & $0.22_{-0.066}^{+0.062}$ & $0.22_{-0.024}^{+0.043}$ \\
\hline $\begin{array}{c}\text { Sample No. } \\
\text { Friction } \\
\text { coefficient f }\end{array}$ & $0.22_{-0.083}^{+0.066}$ & $0.21_{-0.050}^{+0.053}$ & $0.20_{-0.044}^{+0.039}$ \\
\hline
\end{tabular}

From table 3, No. 9 sample has the smallest friction coefficient among all the samples, however, the friction coefficient of smooth blade surface without bionic shark skin is 0.2558 . So bionic shark skin can effectively reduce friction coefficient, and the friction coefficient was reduced by $21.88 \%$

The friction results were also analyzed by orthogonal experimental analysis. The orthogonal experiment results of friction test are shown in table 4 . It can be seen that the impact factors on friction coefficient of bionic shark skins are as follows: riblets pitch (D) $>$ Laser power $(\mathrm{P})>$ Scale length (L). This result implies that the micro riblet structures play the most important role in the friction effect.

Table 4 Orthogonal experiment result of friction test

\begin{tabular}{c|ccc}
\hline & $\mathrm{P} / \mathrm{W}$ & $\mathrm{D} / \mu \mathrm{m}$ & $\mathrm{L} / \mu \mathrm{m}$ \\
\hline $\mathrm{K}_{1}$ & 0.638199 & 0.664491 & 0.64026 \\
$\mathrm{~K}_{2}$ & 0.667569 & 0.631209 & 0.637227 \\
$\mathrm{~K}_{3}$ & 0.635868 & 0.647439 & 0.664149 \\
$\mathrm{k}_{1}\left(=\mathrm{K}_{1} / 3\right)$ & 0.212733 & 0.221497 & 0.21342 \\
$\mathrm{k}_{2}\left(=\mathrm{K}_{2} / 3\right)$ & 0.222523 & 0.210403 & 0.212409 \\
$\mathrm{k}_{3}\left(=\mathrm{K}_{3} / 3\right)$ & 0.211956 & 0.215813 & 0.221383 \\
$\mathrm{R}_{(\mathrm{P}, \mathrm{D}, \mathrm{L})}$ & 0.010567 & 0.011094 & 0.008974 \\
\hline
\end{tabular}

\subsection{Anti-adhesion test}

Finally, fresh liver was cut by electrosurgical knives with bionic shark skins to test the anti-adhesion effect by weighing the adhesive tissue mass on the blades. In the test, cutting speed is $1000 \mathrm{~mm} / \mathrm{min}$ and cutting depth is $12 \mathrm{~mm}$. The temperature of electrosurgical knives was kept to $250^{\circ} \mathrm{C} \pm 10^{\circ} \mathrm{C}$ during the cutting process. After cutting, liver tissue adhesion on the blades were shown in Fig. 7, where Fig. 7(a) shows tissue adhesion on the original blade while
Fig. 7(b) shows tissue adhesion on the blade with bionic shark skin, indicating more tissue adhesion on the original blade. After cutting, electrosurgical knife was cleaned to remove the adhesive tissues. For each electrosurgical knife, cutting experiments were repeated at least 3 times and the average mass of adhesive tissue on the blades with bionic shark skins in table 1 were obtained and shown in table 5 .

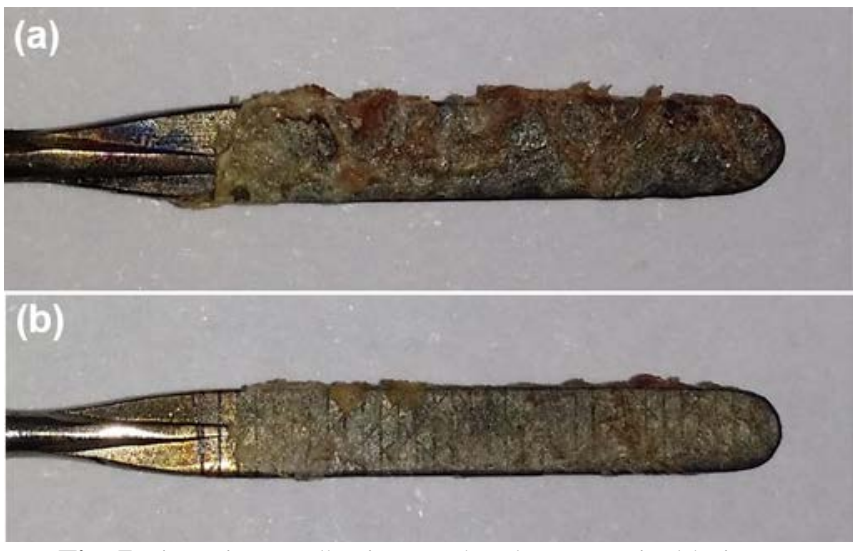

Fig. 7 Liver tissue adhesion on the electrosurgical knives: (a) Original blade without bionic shark skin; (b) The blade with bionic shark skin.

Table 5 Average mass of adhesive tissue on electrosurgical knives

\begin{tabular}{c|ccc}
\hline Sample No. & $\mathbf{1}$ & $\mathbf{2}$ & $\mathbf{3}$ \\
\hline $\begin{array}{c}\text { Adhesive tissue } \\
\text { mass (mg) }\end{array}$ & $2.6_{-0.02}^{+0.02}$ & $2.33_{-0.07}^{+0.05}$ & $2.55_{-0.04}^{+0.03}$ \\
\hline Sample No. & $\mathbf{4}$ & $\mathbf{5}$ & $\mathbf{6}$ \\
\hline $\begin{array}{c}\text { Adhesive tissue } \\
\text { mass (mg) }\end{array}$ & $2.63_{-0.05}^{+0.07}$ & $2.53_{-0.07}^{+0.12}$ & $2.37_{-0.05}^{+0.07}$ \\
\hline Sample No. & 7 & $\mathbf{8}$ & $\mathbf{9}$ \\
\hline $\begin{array}{c}\text { Adhesive tissue } \\
\text { mass (mg) }\end{array}$ & $2.49_{-0.04}^{+0.03}$ & $2.37_{-0.22}^{+0.17}$ & $2.31_{-0.15}^{+0.09}$ \\
\hline
\end{tabular}

As a reference, the average mass of adhesive tissue on the original knife without bionic shark skin is $2.78 \mathrm{mg}$. From table 5, we can conclude that the bionic shark skin can effectively reduce the adhesion of liver tissue and the adhesion mass can be reduced by maximum $16.91 \%$. The anti-adhesion experimental results were also analyzed by orthogonal experimental analysis. The orthogonal experiment results of adhesion test are shown in table 6 . It can be seen that the impact factors affecting the adhesion are as follows: riblets pitch (D)> Laser power (P)> Scale length (L). This result implies that the micro riblet structures also play the most important role in the anti-adhesion performance.

Compared with the results of friction test, the optimal structural parameters and impact factors of adhesion test were consistent. So it is suggested that friction plays a major role in anti-adhesion. Considering surface wettability effect, tissue adhesion decreases with increasing contact angle in the certain range. In total, the smaller friction coef- 
ficient and the greater contact angle, resulting in less adhesion.

Table 6 Orthogonal test results of adhesion test

\begin{tabular}{c|ccc}
\hline & P/W & $\mathrm{D} / \mu \mathrm{m}$ & $\mathrm{L} / \mu \mathrm{m}$ \\
\hline $\mathrm{K}_{1}$ & 7.48 & 7.72 & 7.34 \\
$\mathrm{~K}_{2}$ & 7.53 & 7.23 & 7.27 \\
$\mathrm{~K}_{3}$ & 7.17 & 7.23 & 7.57 \\
$\mathrm{k}_{1}\left(=\mathrm{K}_{1} / 3\right)$ & 2.49 & 2.57 & 2.45 \\
$\mathrm{k}_{2}\left(=\mathrm{K}_{2} / 3\right)$ & 2.51 & 2.41 & 2.42 \\
$\mathrm{k}_{3}\left(=\mathrm{K}_{3} / 3\right)$ & 2.39 & 2.41 & 2.52 \\
$\mathrm{R}_{(\mathrm{P}, \mathrm{D}, \mathrm{L})}$ & 0.12 & 0.16 & 0.1 \\
\hline
\end{tabular}

\section{Conclusions}

In order to minimize the tissue adhesion on the electrosurgical knife during a cutting and hemostasis process, we investigated and fabricated the anti-adhesion microstructures on the blade by fiber laser, inspired by shark skin. 9 blades with different bionic shark skins were fabricated and orthogonal experimental methods were used to find the optimal structural parameters.

The tissue adhesion on the commercial electrosurgical blade made of stainless steel is related to surface wettability and friction coefficient. The surface wettability of samples was studied by measuring the contact angle and experimental results showed that biomimetic shark skin can increase the contact angle and reduce the hydrophilicity. The contact angle can be increased by up to $21 \%$. Orthogonal experimental results implied that the macro structures with scale length $L$ play the most important role in surface wettability.

The friction coefficients of samples were measured by friction and wear testing machine. Friction test results showed that bionic shark skin can effectively reduce friction coefficient and friction coefficient was reduced by $21.88 \%$. Orthogonal experiment results implied that the micro riblet structures play the most important role in the friction effect.

Finally, fresh liver was cut by electrosurgical knives with bionic shark skins to test the anti-adhesion effect by weighing the adhesive tissue mass on the blades by a bal- ance. Orthogonal experiment result implied that the micro riblet structures also play the most important role in the anti-adhesion performance, consistent with the results of friction test, which meant that friction plays a major role in anti-adhesion. Adhesion test results showed that the bionic shark skin can effectively reduce the adhesion of liver tissue and the adhesion mass can be reduced by maximum $16.91 \%$.

\section{Acknowledgments}

This work is funded by National Natural Science Foundation of China (No. 61705124), Opening Fund of State Key Laboratory of Transient Optics and Photonics (No. SKLST201708), Scientific Research Plan Projects of Shaanxi Provincial Education Department (No. 18JK0101) and Doctoral Research Fund from Shaanxi University of Science and Technology (No. 2016BJ-78).

\section{References}

[1] D. Palanker, A. Vankov and P. Jayaraman: New J. Phys., 10, (2008) 123022.

[2] Medtech Insight: US Surgical Procedure Report, No A606, (2007) 1.

[3] A. C. Khandkar and A. A. Hofmann: U. S. Patent 6409725, (2002).

[4] D. B. Brown: J. Vasc. Intervent. Radiol., 16, (2005) 597.

[5] D. Y. Zhang, H. W. Chen, P. F. Zhang and W. W. Zhang: China Patent 104188719A, (2017).

[6] C. C. Lin, H. J. Lin and Y. H. Lin: J. Biomed. Mater. Res. B, 105, (2017) 865.

[7] D. W. Bechert, M. Bruse and W. Hage: Exp. Fluids, 28, (2000) 403.

[8] Y. F. Fu, C. Q. Yuan and X. Q. Bai: Biosurf. Biotrib., 3, (2017) 11.

[9] H. W. Chen, X. Zhang, L. X. Ma, D. Che, D. Y. Zhang and T. S. Sudarshan: Appl. Surf. Sci., 316, (2014) 124.

[10] T. W. Kim: J. Nanosci. Nanotech., 14, (2014) 7562.

[11] X. Chen, T. Li, K Zhai, Z. Hu and M. Zhou: Int. J. Adv. Manu. Tech., 88, (2017) 2727.

[12] A. Marmur: Langmuir, 19, (2003) 8343.

(Received: July 25, 2018, Accepted: November 29, 2018) 